This is the accepted version of the following article: Sol, D. et al "Urbanisation and the loss of phylogenetic diversity in birds" in Ecology letters, vol. 20 (2017), p. 721-9, which has been published in final form at DOI 10.1111/ele.12769. This article may be used for non-commercial purposes in accordance with Wiley Terms and Conditions for SelfArchiving.

\title{
ECOLOGY LETTERS Urbanisation and the loss of phylogenetic diversity in birds
}

\section{7}

Daniel Sol, ${ }^{1,2 *}$ Ignasi

Bartomeus, ${ }^{2}$ Cesar Gonzalez-Lagos ${ }^{3,5}$

and Sandrine Pavoine ${ }^{6}$

\begin{abstract}
Despite the recognised conservation value of phylogenetic diversity, little is known about how it is affected by the urbanisation process. Combining a complete avian phylogeny with surveys along urbanisation gradients from five continents, we show that highly urbanised environments supported on average 450 million fewer years of evolutionary history than the surrounding natural environments. This loss was primarily caused by species loss and could have been higher had not been partially compensated by the addition of urban exploiters and some exotic species. Highly urbanised environments also supported fewer evolutionary distinctive species, implying a disproportionate loss of evolutionary history. Compared with highly urbanised environments, changes in phylogenetic richness and evolutionary distinctiveness were less substantial in moderately urbanised environments. Protecting pristine environments is therefore essential for maintaining phylogenetic diversity, but moderate levels of urbanisation still preserve much of the original diversity.
\end{abstract}

Keywords

Biological invasion, conservation of biodiversity, global change, habitat loss, tolerance to environmental change.

\footnotetext{
${ }^{1}$ CREAF, Cerdanyola del Valles, Catalonia 08193, Spain $\quad{ }^{5}$ Center of Applied Ecology \& Sustainability, Facultad de Ciencias Biologicas, ${ }^{2}$ CSIC, Cerdanyola del Valles, Catalonia 08193, Spain Pontificia Universidad Catolica de Chile, Santiago, Chile

${ }^{2}$ Estacion Biol ogica de Do nana (EBD-CSIC), Sevilla E-41092, Spain

${ }^{3}$ Departamento de Ecologia, Facultad de Ciencias Biologicas, Pontificia Universidad Catolica de Chile, Santiago, Chile

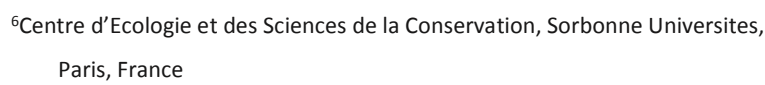




\section{INTRODUCTION}

Urbanisation is currently considered one of the most rapid and drastic alteration of natural ecosystems, causing an important impact on biodiversity and ecosystem services (McKinney 2002; Aronson et al. 2014). Some well-documented consequences include a loss of species richness (e.g. Sol et al. 2014) and an increase in biotic homogenisation (Lockwood et al. 2000; McKinney 2006). Other consequences remain less well studied, however. One is the extent to which urbanisation alters phylogenetic diversity.

Phylogenetic diversity captures the shared ancestry of species both in terms of richness (i.e. amount of evolutionary history) and divergence (i.e. degree at which the species are phylogenetically related; Vellend et al. 2011; Tucker et al. 2016; Veron et al. 2017). Despite the increased appreciation of the intrinsic value of phylogenetic diversity, mainly due to its relevance for preserving evolutionary history (Nee \& May 1997; Purvis et al. 2000; Huang \& Roy 2013; Turley \& Brudvig 2016) and ecosystem functioning (Cadotte et al. 2012), few studies have quantified the phylogenetic consequences of converting natural habitats to cities, particularly at a global scale (Morelli et al. 2016). This is unfortunate considering that future projections predict an increase in urbanised surface by 1.2 million $\mathrm{km}^{2}$ during the first 30 years of the 21 st century (Seto et al. 2012).

The process of urbanisation may affect phylogenetic patterns in a number of ways. One is by reducing phylogenetic richness. As most species do not tolerate well human-driven alterations (Sol et al. 2014), increased urbanisation may decrease phylogenetic richness in parallel with species loss. Yet other consequences are less obvious. If for instance the adaptations needed to thrive in cities are non-randomly distributed across the tree of life, the urbanisation process could lead to a disproportionate loss of evolutionary history (Nee \& May 1997). This loss might result if more vulnerable species are evolutionary distinctive or unique, species lost are clustered within the phylogeny and the phylogeny is imbalanced (Heard \& Mooers 2000; Purvis et al. 2000; Huang et al. 2011; Veron et al. 2017; see also Appendix S1). There is some evidence suggesting a loss of evolutionary distinctiveness in human-altered communities (Fritz \& Purvis 2010; Frishkoff et al. 2014; Morelli et al. 2016), but whether this reflects a higher sensitivity of evolutionary distinct species to urbanisation is less clear.

Urbanisation may alter the phylogenetic diversity of communities in yet another way, by facilitating the entrance of opportunistic species. While most species do not seem to tolerate well the extreme environmental alterations associated with urbanisation, and hence tend to avoid them, some perceive urban environments as ecological opportunities (Blair 1996). These so-called urban exploiters, some of which have an exotic origin, dominate urban communities despite being often absent or rare in the surrounding natural environments (Case 1996; Møller et al. 2015). They thus provide a net gain in the regional phylogenetic richness. Despite this compensatory role, invasions by exotic and native urban exploiters can deeply alter the phylogenetic composition of urban community if they belong to different lineages than the species they replace (Ricciardi \& Atkinson 2004; Li et al. 2015; Sobral et al. 2016). This would be particularly true if, as the Darwin's naturalisation hypothesis suggests, invaders are more successful in communities in which their close relatives are absent (Duncan et al. 2002; Diez et al. 2008; Thuiller et al. 2010).

Here, we combine a full phylogeny of birds (Jetz et al. 2012) with information on well-surveyed assemblages along urbanisation gradients from five continents to address three insufficiently understood questions: (1) How much phylogenetic richness, if any, is lost when natural habitats are urbanised? (2) Is this loss simply the result of species loss or also due to increasing species relatedness and a disproportionate loss of evolutionary distinctive species? And (3) does the presence of native and exotic urban exploiters help compensate the loss of phylogenetic richness? Given the obvious difficulties of conducting a before-after experiment, we addressed these questions by adopting a space-for- 
time substitution approach in which phylogenetic richness and divergence were compared between urbanised environments and the surrounding, little urbanised environments.

\section{METHODS}

Data

We compiled a global data set of species abundances per unit area or unit time for bird assemblages in 27 regions from Europe, North America, Australia, Africa and South America (Appendices S2 and S3). Information came from published studies and our own surveys (Sol et al. 2014). The data included 185908 bird detections belonging to 1219 species, 47 of which were exotic in at least one region.

For each region, spatially defined bird assemblages were available for urbanised environments and the surrounding little urbanised environments ( $n=161$ assemblages; see Data in Appendix S3). To define the degree of urbanisation, we differentiated (1) highly urbanised environments, where buildings were densely packed and parks were small or absent, and (2) moderately urbanised environments, which were residential areas with single-family houses, commonly with backyards (Marzluff et al. 2001). The surrounding, little urbanised environments primarily included woodland (27.6\%), grassland (21.3\%) and rural (34.0\%) habitats, but also habitat mosaics and gardens (17.1\%).

Although the same survey method was used for each habitat within a particular region, comparisons across habitats raise issues about detectability and sampling effort (Lahoz-Monfort et al. 2014). We tackled this by (1) testing whether certain methodological decisions could bias the results, (2) rarefying communities to compare phylogenetic richness with similar sampling effort, (3) taking into account the relative abundance of the species to reduce the influence of rare species, and (4) considering in the models geographic and human-related factors that could influence variation in phylogenetic diversity (see details in the supplementary Methods).

Phylogenetic information

Our analyses were based on a complete, dated phylogeny of birds (Jetz et al. 2012). We dealt with phylogenetic uncertainties in two ways. First, we built a maximum clade credibility phylogeny (CCP) with the TREEANNOTATOR software in BEAST (Drummond et al. 2012) based on 200 phylogenies from the pseudoposterior distribution of 10000 trees available in www.birdtree.org. Second, we repeated the most important analyses with 50 phylogenies randomly chosen from the same pseudoposterior distribution.

Loss of phylogenetic richness in urbanised environments

We used the phylogenies to estimate Faith's phylogenetic diversity (PD, hereafter) for each bird assemblage. PD was measured as the sum of the lengths of all those branches that were members of the corresponding minimum spanning path (Faith 1992). To account for differences in sampling effort among habitats, we also calculated PD rarefied by randomly sampling individuals from the regional pool; the sample size was the minimum community size among all assemblages within a region (Cardoso et al. 2015). To assess the influence of rare species on phylogenetic diversity measures (Fine \& Kembel 2011), we used a recently proposed phylogenetic diversity metric based on Hill numbers (PDw hereafter) that is sensitive to both species abundance and phylogenetic distances (Chao et al. 2010). We set the $\mathrm{q}$ parameter to 1, meaning that a species weight in the Hill number was proportional to its abundance.

To assess whether the loss of phylogenetic richness merely resulted from species loss, we standardised PD and PDw (PDI and PDIw, hereafter) by subtracting from the observed values the mean values expected under a null model in 
which taxa labels were shuffled across the tips of the regional phylogeny 999 times. This null model generates randomised phylogenetic relationships among taxa while maintaining the observed phylogenetic tree, species abundance distribution, and community structure. The effect size was then divided by the standard deviation of the randomised values. The more negative PDI and PDIw, the more phylogenetically clustered species were locally compared to the regional species pool.

All the above metrics were estimated for each assemblage with the R-packages "Picante" (Kembel et al. 2010) and "BAT" (Cardoso et al. 2015) as well as our own scripts. Differences across urbanised and little urbanised environments were then evaluated by means of linear mixed models, using the Bayesian approximation implemented in the Rpackage 'MCMCgImm' (Hadfield 2010). As the response variables were continuous and exhibited "bell curve" shapes, we used models with a Gaussian structure of errors. Region was included as random effect to allow phylogenetic richness to be compared within urbanisation gradients. To account for spatial autocorrelation, region was nested within country and in some models we included geographic longitude and/or distance to Equator as fixed effects (see Appendix S4 for justifications). The survey method, sampling effort and whether detectability was or was not taken into account were also included as fixed effects, along with season, altitude, urbanisation period and urban area (see also Sol et al. 2014). To check for model convergence, we ran the model twice with different starting values, sampling 1000 iterations from a total of 1100000 . When the model did not adequately converge, we sequentially increased the total number of iterations and number of iterations passed before samples were stored. We further explored the influence of species loss on PD decline by means of Piecewise structural equation modelling (Lefcheck 2015).

Changes in phylogenetic composition with urbanisation

To quantify dissimilarity in phylogenetic composition among urbanised and the surrounding little urbanised environments, we estimated UniFrac b-diversity indices, a derivation of the Jaccard dissimilarity index that quantifies the proportion of shared branch length between pairs of assemblages (Leprieur et al. 2012). We compared the indices for each combination of habitats by means of the MCMCgImm approach outlined above. Then, we separated 'True' turnover of lineages (turnover, hereafter) from phylogenetic diversity gradients (nestedness), following Leprieur et al. (2012). A predominance of turnover over nestedness would imply that b-diversity does not merely reflect a reduction in diversity but a different combination of phylogenetic lineages.

As we detected substantial turnover, we estimated the fraction of PD coming from exploiters (see definitions below), exotics and non-exploiters. To this purpose, we estimated the evolutionary distinctiveness (ED) of the three groups based on subtrees only containing the species of the assemblage. ED was calculated as the weighted sum of the edge lengths from the root to a tip of the tree, with the weights being 1 /number of species that share that edge (Isaac et al. 2007). Following Sol et al. (2014), we operationally defined an exploiter as a native species that was more abundant in the urbanised environment than expected by their abundance in the little urbanised surrounding environments (see Blair et al. 2001 for a more general definition). We also used a second, less restrictive classification in which we also considered exploiter those species that despite exhibiting abundances lower than expected by chance were still abundant in highly urbanised environment. We used a threshold of relative abundance $>5 \%$, but the use of a higher threshold did not alter the conclusions.

Phylogenetic effects of urbanisation tolerance

Using the distinction between exploiter and non-exploiter, we investigated phylogenetic effects in the tolerance of species to urbanisation in two ways. First, we measured its phylogenetic signal by means of the D statistic (Fritz \& Purvis 2010), as implemented in the R-package Caper (Orme et al. 2013). Second, we tested whether some avian families had more or less exploiters than expected by chance by means of randomisations, as described in the 
Supplementary methods. We illustrate the results with phylogenetic reconstructions based on the functions "contMap" and "plotSimmap" from the Phytools R-package (Revell 2011).

Evolutionary distinctiveness loss with urbanisation tolerance

To assess whether urban communities maintained less phylogenetically distinctive species, we again calculated the weighted sum of the edge lengths from the root to a tip of the tree (Isaac et al. 2007). In this case, however, ED was estimated based on the entire phylogeny of 9993 avian species (EDg, hereafter). EDg of each assemblage was estimated as the average value for all species present and modelled as a function of habitat with the Gaussian MCMCgImm approach outlined in previous sections. To help interpret differences in EDg, we also estimated evolutionary uniqueness (EU), measured as the length of the terminal branch of the species.

Using again the distinction between exploiter and nonexploiter, we finally asked whether vulnerable species fall within species poor, phylogenetically isolated clades by comparing EDg between urban exploiters and avoiders by means of a binomial MCMCgImm. Because being an exploiter does not only depend on the features of the species itself but also on how it interacts with other species from the region, we conducted the analyses at the assemblage level and included region and country as random factors in a nested structure together with the species identity and phylogeny.

\section{RESULTS}

Loss of native phylogenetic richness in urbanised environments

Highly urbanised environments supported on average 458.7346 .8 million fewer years of evolutionary history than the surrounding little urbanised environments (pMCMC < 0.001; Figs 1 and 2, S1 and S2; Tables S1 and S2). A decline in PD was also observed when considering the relative abundance of species, yet in this case the decline was less pronounced (Fig. 1, Tables S3). Compared with highly urbanised environments, the loss of PD in moderately urbanised environments relative to surrounding environments was less dramatic (211.4 398.4 millions of years; Fig. 1, Tables S1-S3).

The observed decline in PD with urbanisation was robust to the effect of phylogenetic uncertainties (Fig. S3) and a set of potentially confounding variables such as urbanisation period, urbanised area and distance to the Equator (see Tables S1-S3). Moreover, the decline was highly consistent regardless of the nature of the surrounding little-urbanised habitats (Fig. S4). Although the decline in PD was more accentuated in some regions than in others, we did not detect any clear latitudinal or longitudinal trend (Appendix S4). The amount of PD loss in a region was also unrelated to species richness or the duration and extension of the urban alterations (Appendix S4).

Mitigation of PD loss by the addition of native urban exploiters

Differences in phylogenetic b-diversity were particularly important in transitions from little to highly urbanised environments (Table S4, Figs 3, S5). In these transitions, there was a predominance of turn-over relative to nestedness (Figs 3, S5), reflecting the existence of some species that were only present in urbanised environments. In many regions, exploiters explained a substantial fraction of total variation in PD (Fig. S6).

Native species richness as a driver of PD loss with urbanisation

The net loss of PD with urbanisation may result of two processes (see Appendix S1): species loss and increasing species 


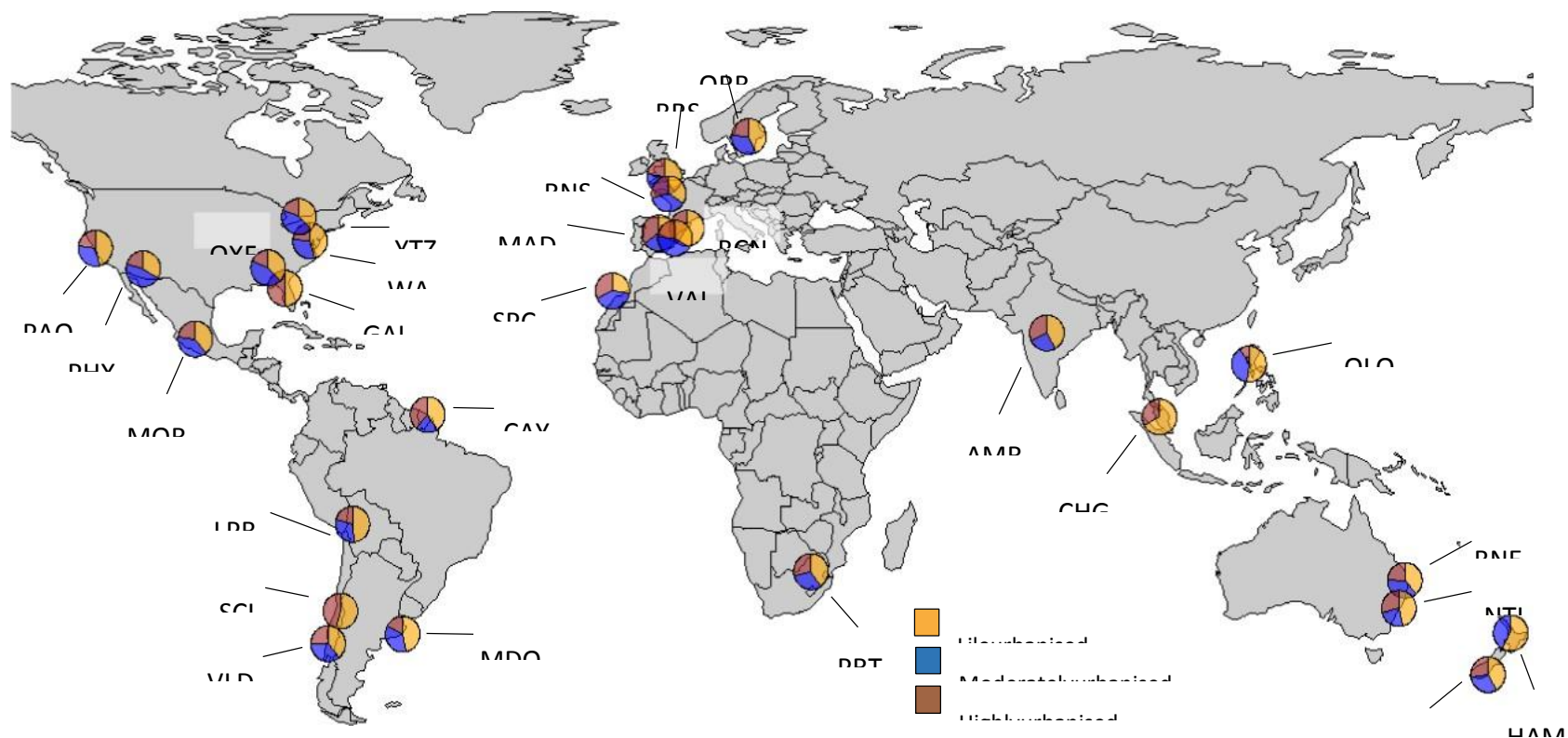

niln

Figure 1 Worldwide location of the study regions, showing the proportion of Faith's phylogenetic diversity (PD) according to the degree of urbanisation. The abbreviations for the regions are in the Appendix S2. Note that not all the regions contain all the habitats.
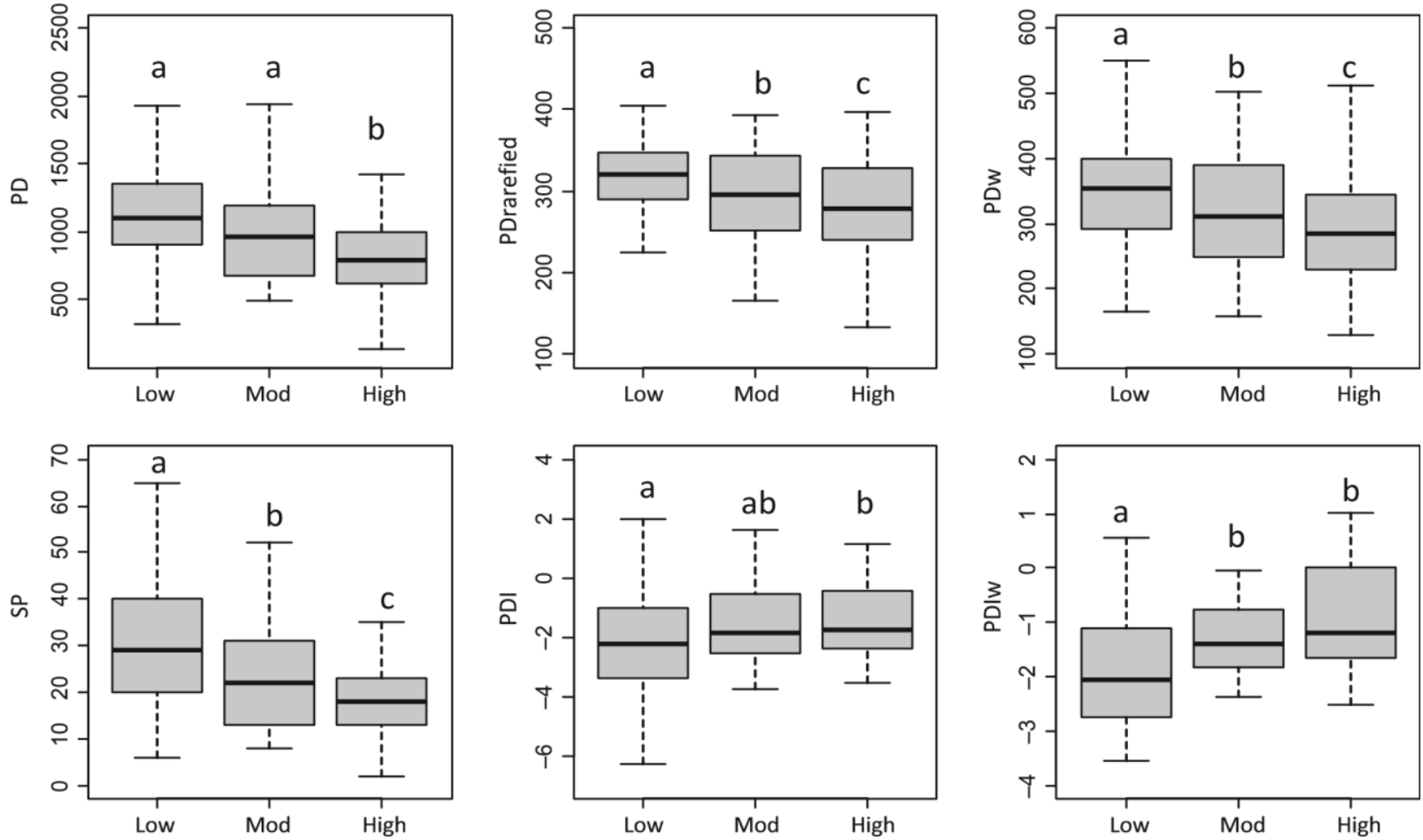

Figure 2 Differences in phylogenetic richness (PD, in million years), rarefied phylogenetic richness (PDrarefied), phylogenetic richness taking into account species relative abundance (PDw), species richness (SP), and standardised effect sizes of PD (PDI) and PDw (PDIw) among little urbanised (low), moderately urbanised (mod) and highly-urbanised (high) environments for native species. Letters indicate differences among habitats at $\mathrm{P}<$ 0.05 (see Tables S1 and 3, S5 and 6 for full models), examined by changing the level of reference with which the other habitats are compared. 
relatedness (Frishkoff et al. 2014). We found clear evidence exploiters, native species richness in highly urbanised environfor a main role of species loss. Even when considering the ments was on average over two times lower than that of the gain in species due to the entrance of obligate native surrounding little urbanised environments (Fig. 2). Once
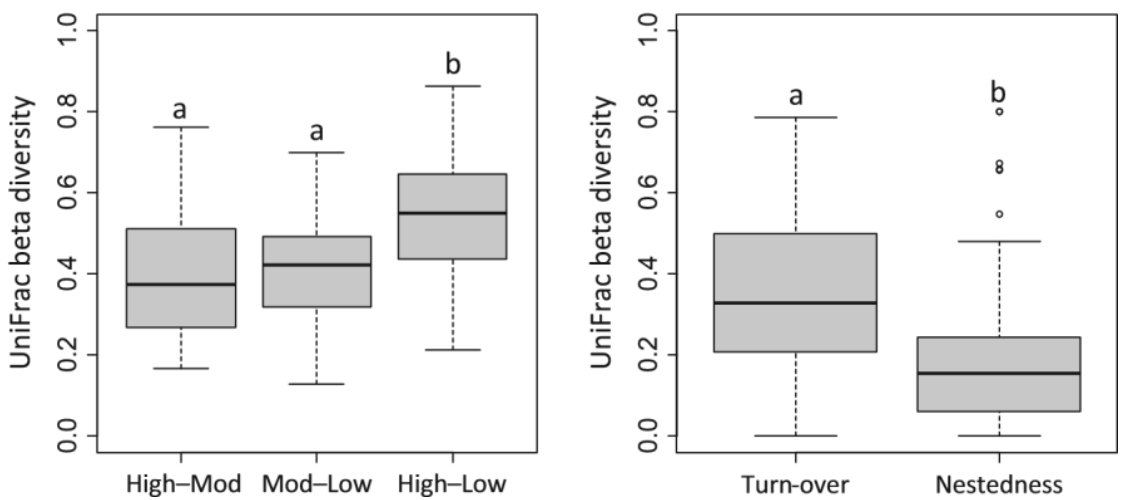

Figure 3 UniFrac b-diversity index comparing differences between different habitats (left panel) and how is affected by turnover and nestedness when comparing highly and little urbanised environments (right panel). High = highly-urbanised, Mod = moderately-urbanised, Low = little urbanised; see Table S4 for formal analyses.

phylogenetic richness was standardised by estimating PDI and PDIw, phylogenetic richness no longer decreased with urbanisation but increased (Fig. 2, Tables S5 and S6). Piecewise structural equation modelling further confirmed the influence of species richness, showing that in the best supported causal scenarios habitat affected PD indirectly through its influence on species richness (Fig. S7).

Phylogenetic effects in tolerance to urbanisation

Urban exploiters were more likely to be found in certain clades, as reflected by the existence of phylogenetic signal (Fig. S8). Although a wide array of clades across the avian phylogeny thrived in highly urbanised environments (Figs 4 and 59), urban exploiters tended to be overrepresented in a few distantly-related families that exhibit relatively low EDg (Table S7; Fig. 4). Some of these families also contained highly successful invaders (Table S8).

Loss of evolutionary distinctiveness with urbanisation.

Mean evolutionary distinctiveness (EDg) of native species assemblages tended to be lower in highly urbanised environments compared to little urbanised environments (pMCMC $<0.001$ in all cases; Table S9, Fig. 5). This largely reflected the effect of evolutionary uniqueness (Table S10), a metric strongly correlated with EDg (Fig. 5). The decrease in EDg with urbanisation was in part the result of a decline in species richness (Fig. S7). However, it also reflected a lower tolerance of evolutionary distinctive species to urbanisation. Species 

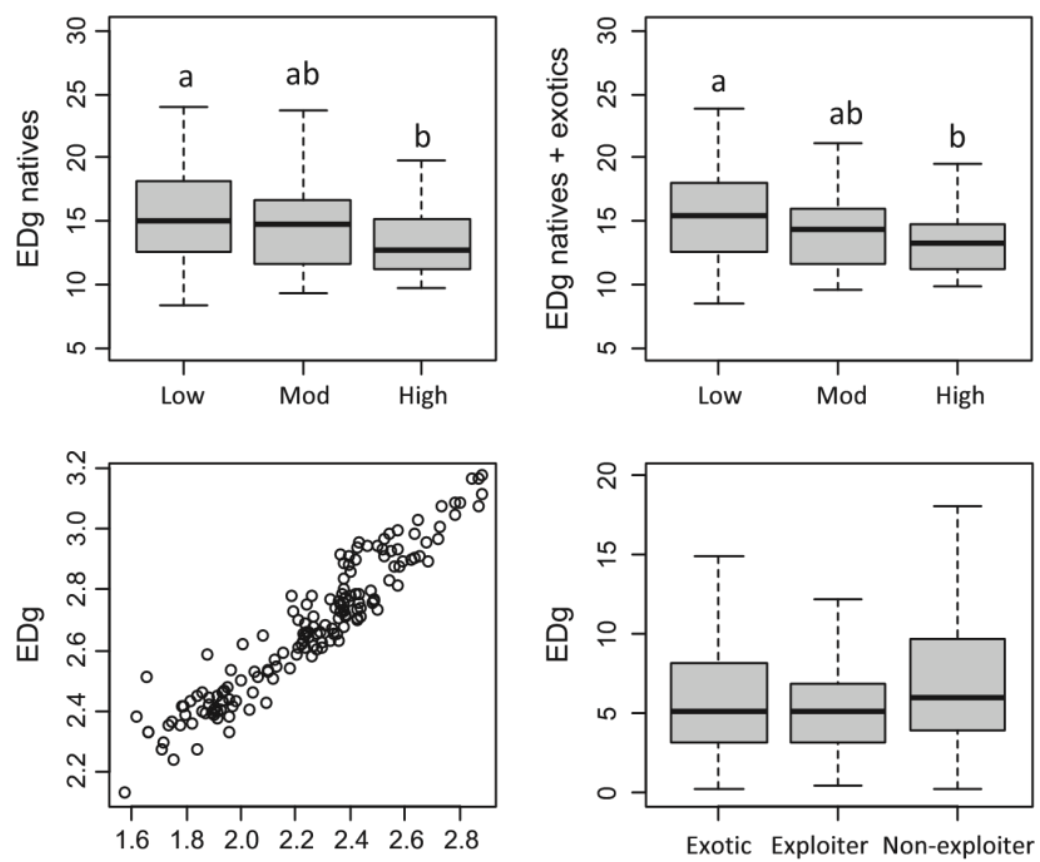

EU

Exotic Exploiter Non-exploiter

Figure 4 Left panel, family level variation in the likelihood to contain urban exploiters. Values close to 0 indicate that the family has more urban exploiters than expected by chance whereas values close to 1 suggest that the family has less exploiters than expected by chance. States of internal nodes are Maximum Likelihood reconstructions as implemented in the function "contMap" of Phytools (Revell 2011). Only families with 15 or more species are presented. For more details, see Table S7. Right panel, evolutionary distinctiveness (EDg) averaged for all the species of each family and standardised by substracting the EDg averaged across all avian families. Confidence intervals represent standard deviations. Values below zero indicate that the family has an EDg lower than the mean estimated for all the avian families.
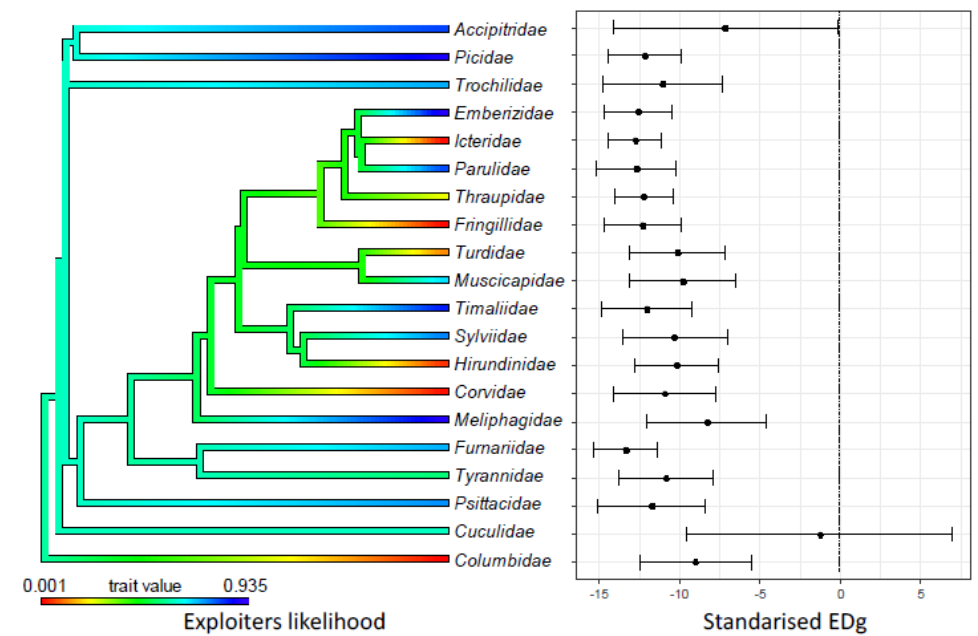

Figure 5 Above, community mean differences in evolutionary distinctiveness (EDg), among little urbanised (low), moderately-urbanised (mod) and highlyurbanised (high) environments, considering or not exotic species (see Tables S9 and S13). Below, relationship between EDg and EU across species (left) and differences in EDg among native exploiter, native non-exploiter and exotic species (see Tables S11 and S12 and main text). In all cases, EDg has been estimated based on the complete phylogeny of all extant bird species (Jetz et al. 2012). Letters indicate differences among habitats at $\mathrm{P}<0.05$. 
with lower EDg were more likely to be urban exploiters (pMCMC $<0.001$ in all cases; Tables S11 and S12, Fig. 5). Although EDg co-varied with geographic longitudes (Tables S9 and S10, see also Jetz et al. 2012), the loss of EDg with urbanisation was largely independent of this effect (Tables S11 and S12). The decline in EDg with urbanisation was more accentuated in regions with higher mean community evolutionary distinctiveness (Appendix S4). Other factors like species richness, urbanisation period, urban area, geographical longitude and distance to Equator had no effect on how much EDg was lost (Appendix S4).

Compensation of phylogenetic diversity by exotics in urbanised environments

Exotic species did not fully compensate for the loss of PD and EDg associated with urbanisation. Despite that exotic species tended to attain higher success in urbanised habitats (Table S8), the loss of PD and EDg with urbanisation was still substantial when including exotic birds in the analyses (Figs S6 and S10; Table S13). This in part resulted from the reduced number of exotic species present (range 0-17). In places where this number was high, like in New Zealand, the compensatory effect was more substantial (see Fig. S6). However, the low compensatory effect of exotic species also reflected that these species were not more evolutionary distinctive than the native species they replaced (Binomial MCMCgImm: $\mathrm{P}=0.65$ and 0.63 , depending on the tolerance metric, Fig. 5).

\section{DISCUSSION}

Despite the growing concern over the impact of rapid humaninduced environmental changes on biodiversity, assessments of how the conversion of natural habitats to cities affects phylogenetic diversity have been rare (but see Morelli et al. 2016). Our comprehensive global analysis contributes to fill this gap by showing that urbanisation generally causes a significant decline in phylogenetic richness, an impact that varies across regions. The decline increased with the intensity of the urbanisation and was highly consistent regardless of the nature of the surrounding little urbanised habitats. Rarefactions suggest that the pattern was not merely the result of smaller community sizes in urbanised environments. However, phylogenetic richness loss was less pronounced when considering the relative abundance of species, perhaps reflecting phylogenetic redundancies in urbanised environments and/or that the loss was partially compensated by an increase in abundance evenness across the phylogeny.

By homogenising the physical environment to meet the demands of human beings, cities may be creating filters that determine that only some lineages can persist in these new environments (McKinney 2006). Our results confirm this expectation, showing that tolerance to urbanisation was clustered within the phylogeny (see also Sol et al. 2014). Thus, although a wide array of clades across the avian phylogeny thrived in highly urbanised environments, urban exploiters tended to be overrepresented in a few families. These include species-rich, widely distributed families like pigeons, crows, finches, swallows, and grackles, which are often represented by one or a few species in a given region but by distinct species in different regions. In pigeons, for example, urban representatives include speckled pigeons (Columba guinea) in Africa, Collared Doves (Streptopelia decaocto) in Eurasia, Crested pigeons (Ocyphaps lophotes) in Australia, and Zenaida doves (Zenaida aurita) in the Caribbean. The homogenisation of the physical environment of cities results thus in increased phylogenetic homogenisation (McKinney 2006).

Even though urban exploiters were phylogenetically clustered in the tree of life, there was no evidence that this significantly contributed to the reduced phylogenetic richness of urban communities. Rather, most of the observed loss of phylogenetic richness associated with urbanisation was explained by species loss. Once standardised with values obtained from random expectations, phylogenetic diversity no longer decreased with urbanisation but increased. This implies that urban communities tended to have more phylogenetic richness than expected by the number of species present, notably when the intensity of urbanisation was high. The pattern may be interpreted as species in urbanised 
environments being more distantly related to other species, or at least equally distantly related, than species from natural, surrounding communities.

Despite sustaining a higher phylogenetic richness than expected by species richness, the average evolutionary distinctiveness of birds in highly urbanised environments tended to be lower compared to little urbanised environments. Morelli et al. (2016) also found that urbanisation caused an important loss of avian evolutionary distinctiveness in European cities. The loss of evolutionary distinctiveness was primarily driven by the loss of pendant edges, a pendant edge being the terminal branch that connects a species to the rest of the tree (see also Redding et al. 2014), and was higher in regions that held more evolutionary distinctive species. Therefore, it is possible that the loss of phylogenetic diversity has been sub-estimated by including in our analyses regions where most evolutionary distinctive species were extirpated a long time ago.

Unlike differences in phylogenetic richness, the decrease in the mean community evolutionary distinctiveness with urbanisation was not merely the result of a decline in species richness. Rather, it also reflected a lower tolerance of evolutionary distinctive species to urbanisation, in line with the overrepresentation of threatened species in poorly diversified clades (Bennett \& Owens 1997). While the mechanisms remain unclear, one possible explanation is that these species belong to clades that have historically had difficulties to cope with environmental changes, and hence may have experienced high extinction rates and/or reduced speciation rates.

Simulations show that a reduction in evolutionary distinctiveness has little impact on phylogenetic richness if not accompanied by phylogenetic imbalance and by the phylogenetic clustering of species loss (Veron et al. 2017). Thus, a loss of evolutionary distinctive species does not necessarily imply a loss of phylogenetic richness. Still, the finding that evolutionary distinctive species often exhibit lower tolerance to urbanisation suggests that transforming natural habitats to cities can have an impact on the tree of life (see also Frishkoff et al. 2014).

The observed loss of phylogenetic richness in cities could have been more important if it was not for the arrival of a number of urban exploiters, which dominated these communities despite being rare or absent in the surrounding little urbanised environments. As these "obligate" urban exploiters tended to be over-represented in a few distantly related clades and often lacked close-relatives in the same community, their presence may contribute to explain why highly urbanised environments had a higher phylogenetic richness than expected by the number of species they sustained. Birds successfully thriving in urbanised environments therefore represent to some extent novel communities featuring a small number of distantly related taxa from little evolutionary distinctive clades. Our findings support thus claims that the ongoing global changes driven by human activities do not merely reduce biodiversity but also lead to the emergence of novel communities with different phylogenetic and functional properties (Lurgi et al. 2012).

Although exotic species tended to concentrate in urbanised habitats (see Sol et al. 2016), their contribution to increasing phylogenetic richness was quite modest. This is because the number of exotics species within the studied regions was generally low. Moreover, exotic species tended to belong to little evolutionary distinctive clades. Thus, the replacement of native species by exploiters and exotics can maintain or even increase taxonomic diversity, but nevertheless reduce part of the evolutionary history of the community (Sobral et al. 2016; but see Garcia et al. 2014). As exotic birds are overrepresented in a few clades (Blackburn \& Duncan 2001), they should also contribute to increase phylogenetic homogenisation of urbanised environments.

Our global analysis provides evidence that high levels of urbanisation reduce and alter phylogenetic diversity, favouring a few species with many close relatives at the expense of species from more evolutionary distinct clades. Phylogenetic diversity has intrinsic conservation value because it does not only take into account species richness but also captures genetic diversity, ecosystem functionality and evolutionary history (Faith 1992; Nee \& May 1997; Purvis et 
al. 2000; Huang et al. 2011). While preserving little urbanised environments is crucial for maintaining phylogenetic diversity across the tree of life, our results are in line with Frishkoff et al. (2014) that moderate intensities of environmental alterations may protect against extreme loss of phylogenetic diversity.

\section{ACKNOWLEDGEMENTS}

We thank all authors who published the information on their intensive urban avian surveys, without which this study could not have been possible. We are also in debt with Joan GarciaPorta for generating the CCP and Dario Moreira for helping assemble the data set. We finally thank Phillip Clergeau, Mariana Villegas and Ivan Diaz for providing unpublished data, Marı Moiron, Gabriel Garcıa-Pe na, Miquel Vall-llosera, Louis Lefebvre and three anonymous reviewers for insightful comments, Walter Jetz and co-workers for making available their phylogeny of birds, and the Rteam and package contributors for the $R$ free software. This paper is part of the project CGL2013-47448-P from the Spanish Government to DS and IB. CG-L was supported by the grant PUC1203

MECESUP-Pontifica Universidad Catolica de Chile.

\section{DATA ACCESSIBILITY STATEMENT}

Data are available as supplementary material.

\section{AUTHORSHIP}

DS conceived the study, CGL and DS gathered data, DS analysed the data with contributions of IB, CGL and SP, DS wrote the paper, and all authors contributed to the different drafts of the paper.

\section{REFERENCES}

Aronson, M.F.J., La Sorte, F.A., Nilon, C.H., Katti, M., Goddard, M.A., Lepczyk, C.A. et al. (2014). A global analysis of the impacts of urbanization on bird and plant diversity reveals key anthropogenic drivers. Proc. R. Soc. Lond. B, 281, 20133330

Bennett, P.M. \& Owens, I.P.F. (1997). Variation in extinction risk among birds: chance or evolutionary predisposition? Proc. R. Soc. B Biol. Sci., 264, 401-408.

Blackburn, T.M. \& Duncan, R.P. (2001). Establishment patterns of exotic birds are constrained by non-random patterns in introduction. J. Biogeogr., 28, 927-939.

Blair, R.R.B. (1996). Land use and avian species diversity along an urban gradient. Ecol. Appl., 6, 506-519.

Blair, R.B. (2001). Birds and butterflies along urban gradients in two ecoregions of the U.S. In: Biotic Homogenization (eds Lockwood, J.L. \& McKinney, M.L.). Book Section. Kluwer, Norwell, MA, pp. 33-56

Cadotte, M.W., Dinnage, R. \& Tilman, D. (2012). Phylogenetic diversity promotes ecosystem stability. Ecology, 93, S223-S233.

Cardoso, P., Rigal, F. \& Carvalho, J.C. (2015). BAT - biodiversity assessment tools, an R package for the measurement and estimation of alpha and beta taxon, phylogenetic and functional diversity. Methods Ecol. Evol., 6, 232-236.

Case, T.J. (1996). Global patterns in the establishment and distribution of exotic birds. Biol. Conserv., 78, 69-96.

Chao, A., Chiu, C.-H. \& Jost, L. (2010). Phylogenetic diversity measures based on Hill numbers. Philos. Trans. R. Soc. Lond. B Biol. Sci., 365, 35993609.

Diez, J.M., Sullivan, J.J., Hulme, P.E., Edwards, G. \& Duncan, R.P. (2008). Darwin's naturalization conundrum: dissecting taxonomic patterns of species invasions. Ecol. Lett., 11, 674-681.

Drummond, A.J., Suchard, M.A., Xie, D. \& Rambaut, A. (2012). Bayesian phylogenetics with BEAUti and the BEAST 1.7. Mol. Biol. Evol., 29, 19691973. 
Duncan, R.P., Williams, P.A., Wikelski, M., Wong, V., Chevalier, B., Rattenborg, N. et al. (2002). Darwin's naturalization hypothesis challenged. Nature, 417, 608-609.

Faith, D.P. (1992). Conservation evaluation and phylogenetic diversity. Biol. Conserv., 61, 1-10.

Fine, P.V.A. \& Kembel, S.W. (2011). Phylogenetic community structure and phylogenetic turnover across space and edaphic gradients in western Amazonian tree communities. Ecography (Cop.), 34, 552-565.

Frishkoff, L.O., Karp, D.S., M'Gonigle, L.K., Mendenhall, C.D., Zook, J., Kremen, C. et al. (2014). Loss of avian phylogenetic diversity in neotropical agricultural systems. Science (80-.)., 345, 1343-1346

Fritz, S.A. \& Purvis, A. (2010). Selectivity in mammalian extinction risk and threat types: a new measure of phylogenetic signal strength in binary traits. Conserv. Biol., 24, 1042-1051.

Garcia, D., Martinez, D., Stouffer, D.B. \& Tylianakis, J.M. (2014). Exotic birds increase generalization and compensate for native bird decline in plantfrugivore assemblages. J. Anim. Ecol., 83, 1441-1450.

Hadfield, J.D.J. (2010). MCMC methods for multi-response generalized linear mixed models: the MCMCgImm R package. J. Stat. Softw., 33, $1-22$.

Heard, S.B. \& Mooers, A.O. (2000). Phylogenetically patterned speciation rates and extinction risks change the loss of evolutionary history during extinctions. Proc. R. Soc. Lond. B, 267, 613-620.

Huang, D. \& Roy, K. (2013). Anthropogenic extinction threats and future loss of evolutionary history in reef corals. Ecol. Evol., 3, $1184-1193$.

Huang, S., Davies, T.J. \& Gittleman, J.L. (2011). How global extinctions impact regional biodiversity in mammals. Biol. Lett., 8, $222-225$.

Isaac, N.J.B., Turvey, S.T., Collen, B., Waterman, C. \& Baillie, J.E.M. (2007). Mammals on the EDGE: conservation priorities based on threat and phylogeny. PLoS ONE, 2, e296.

Jetz, W., Thomas, G., JB, J., Hartmann, K. \& Mooers, A. (2012). The global diversity of birds in space and time. Nature, 491, 444-448

Kembel, S.W., Cowan, P.D., Helmus, M.R., Cornwell, W.K., Morlon, H., Ackerly, D.D. et al. (2010). Picante: R tools for integrating phylogenies and ecology. Bioinformatics, 26, 1463-1464.

Lahoz-Monfort, J.J., Guillera-Arroita, G. \& Wintle, B.A. (2014). Imperfect detection impacts the performance of species distribution models. Glob. Ecol. Biogeogr., 23, 504-515.

Lefcheck, J.S. (2015). piecewiseSEM : piecewise structural equation modeling in R for ecology, evolution, and systematics. Methods Ecol. Evol., 7, 573-579.

Leprieur, F., Albouy, C., deBortoli, J., Cowman, P.F., Bellwood, D.R. \& Mouillot, D. (2012). Quantifying phylogenetic beta diversity: distinguishing between "true" turnover of lineages and phylogenetic diversity gradients. PLoS ONE, 7, e42760.

Li, S.P., Cadotte, M.W., Meiners, S.J., Hua, Z.S., Shu, H.Y., Li, J.T. et al. (2015). The effects of phylogenetic relatedness on invasion success and impact: deconstructing Darwin's naturalisation conundrum. Ecol. Lett., 18, 1285-1292.

Lockwood, J.L., Brooks, T.M. \& Mckinney, M.L. (2000). Taxonomic homogenization of the global avifauna. Anim. Conserv., 3, 27-35.

Lurgi, M., Lopez, B.C. \& Montoya, J.M. (2012). Novel communities from climate change. Philos. Trans. R. Soc. Lond. B Biol. Sci., 367, $2913-2922$.

Marzluff, J.M., Bowman, R. \& Donnelly, R. (2001). A historical perspective on urban bird research: trends, terms, and approaches. In: Avian ecology and conservation in an urbanized world (eds Marzluff, J.M., Bowman, R. \& Donnelly, R.). Kluwer Academic, Boston, pp. 1-17.

McKinney, M.L. (2002). Urbanization, biodiversity and conservation. Bioscience, 52, 883-890.

McKinney, M. (2006). Urbanization as a major cause of biotic homogenization. Biol. Conserv., 127, 247-260.

Møller, A.P., Dıaz, M., Flensted-Jensen, E., Grim, T., Ibanez- A lamo, J.D., Jokim€aki, J. et al. (2015). Urbanized birds have superior establishment success in novel environments. Oecologia, 178, 943-950.

Morelli, F., Benedetti, Y., Ibanez- Alamo, J.D., Jokimaki, J., M €and, R., Tryjanowski, P. et al. (2016). Evidence of evolutionary homogenization of bird communities in urban environments across Europe. Glob. Ecol. Biogeogr., 1-10.

Nee, S. \& May, R.M. (1997). Extinction and the Loss of Evolutionary History. Science, 278, 692-694.

Orme, D., Freckleton, R., Thomas, G., Petzoldt, T., Fritz, S., Isaac, N. et al. (2013). Caper: Comparative Analyses of Phylogenetics and Evolution in R. R Packag. version 0.5.2., https://CRAN.R-project.org/ package=caper

Purvis, A., Agapow, P.M., Gittleman, J.L. \& Mace, G.M. (2000). Nonrandom extinction and the loss of evolutionary history. Science, 288, 328-330

Redding, D.W., Mazel, F. \& Mooers, A. (2014). Measuring evolutionary isolation for conservation. PLoS ONE, 9, e113490. 
Revell, L.J. (2011). Phytools: an R package for phylogenetic comparative biology (and other things). Methods Ecol. Evol., 3, $217-223$.

Ricciardi, A. \& Atkinson, S.K. (2004). Distinctiveness magnifies the impact of biological invaders in aquatic ecosystems. Ecol. Lett., 7, $781-784$.

Seto, K.C., Guneralp, B. \& Hutyra, L.R. (2012). Global forecasts of€ urban expansion to 2030 and direct impacts on biodiversity and carbon pools. Proc. Natl Acad. Sci. USA, 109, 16083-16088.

Sobral, F.L., Lees, A.C. \& Cianciaruso, M.V. (2016). Introductions do not compensate for functional and phylogenetic losses following extinctions in insular bird assemblages. Ecol. Lett., 19, 1091-1100.

Sol, D., Gonzalez-Lagos, C., Moreira, D., Maspons, J. \& Lapiedra, O. (2014). Urbanisation tolerance and the loss of avian diversity. Ecol. Lett., 17, 942950.

Sol, D., Gonzalez-Lagos, C., Lapiedra, O. \& Dıaz, M. (2016) Why are exotic birds so successful in urbanized environments? In: Urban Ornithology (ed Murgui, H.), Springer, Switzerland, pp 75-89.

Thuiller, W., Gallien, L., Boulangeat, I., De Bello, F., Munkem€ uller, T.,€ Roquet, C. et al. (2010). Resolving Darwin's naturalization conundrum: a quest for evidence. Divers. Distrib., 16, 461-475.

Tucker, C.M., Cadotte, M.W., Carvalho, S.B., Davies, T.J., Ferrier, S., Fritz, S.A. et al. (2016). A guide to phylogenetic metrics for conservation, community ecology and macroecology. Biol. Rev., 10. 1111/brv.12252, in press

Turley, N.E. \& Brudvig, L.A. (2016). Agricultural land-use history causes persistent loss of plant phylogenetic diversity. Ecology, 97, $2240-2247$.

Vellend, M., Cornwell, W.K., Magnuson-ford, K. \& Mooers, A.Ø. (2011). Measuring phylogenetic biodiversity. In Biological Diversity: Frontiers in Measurement and Assessment (eds Magurran, A.E., McGill, B.J.). Oxford University Press, Oxford.

Veron, S., Davies, T.J., Cadotte, M.W., Clergeau, P. \& Povaine, S. (2017). Predicting loss of evolutionary history: Where are we? Biol.

Rev., 92, 271-291.

\section{SUPPORTING INFORMATION}

Additional Supporting Information may be found online in the supporting information tab for this article. 